CARDIOVASCULAR MEDICINE

\title{
Time to treatment and the impact of a physician on prehospital management of acute ST elevation myocardial infarction: insights from the ASSENT-3 PLUS trial
}

\author{
R C Welsh, W Chang, P Goldstein, J Adgey, C B Granger, F W A Verheugt, \\ L Wallentin, F Van de Werf, P W Armstrong, on behalf of the ASSENT-3 PLUS \\ Investigators
}

See end of article for authors' affiliations

Correspondence to: Dr Robert C Welsh, University of Alberta, 2C2 Walter C Mackenzie Health Sciences Centre, Edmonton, Alberta, Canada T6G 2B7; rwelsh@cha.ab.ca

Accepted 2 February 2005 Published Online First 17 March 2005

\begin{abstract}
Objectives: To assess the impact of variation in prehospital care across distinct health care environments in ASSENT (assessment of the safety and efficacy of a new thrombolytic) -3 PLUS, a large ( $n=1639$ ) contemporary multicentred international trial of prehospital fibrinolysis. Specifically, the objectives were to assess predictors of time to treatment, whether components of time to treatment vary across countries, and the impact of physician presence before hospitalisation on time to treatment, adherence to protocol, and clinical events.

Methods: Patient characteristics associated with early treatment $(\leqslant 2$ hours), comparison of international variation in time to treatment, and components of delay were assessed. Trial specific patient data were linked with site specific survey responses.

Results: Younger age, slower heart rate, lower systolic blood pressure, and prior percutaneous coronary intervention were associated with early treatment. Country of origin accounted for the largest proportion of variation in time. Intercountry heterogeneity was shown in components of elapsed time to treatment. Physicians in the prehospital setting enrolled $63.8 \%$ of patients. The presence of a physician was associated with greater adherence to protocol mandated treatments and procedures but with delay in time to treatment $(120 \vee 108$ minutes, $\mathrm{p}<0.001)$.

Conclusion: Country of enrolment accounted for the largest proportion of variation in time to treatment and intercountry heterogeneity modulated components of delay. The effectiveness and safety of prehospital fibrinolysis was not influenced by the presence of a physician. These data, acquired in diverse health care environments, provide new understanding into the components of prehospital treatment delay and the opportunities to further reduce time to fibrinolysis for patients with ST elevation myocardial infarction.
\end{abstract}

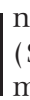
patients with acute ST elevation myocardial infarction (STEMI), time to treatment is a consistent and salient modulator of successful reperfusion and improved patient outcomes. Despite educational interventions with patients and physicians to reduce temporal delay, registry and clinical trial data continue to show suboptimal times to treatment with pharmacological and mechanical reperfusion strategies. $^{12}$ Prehospital fibrinolysis has been shown to significantly reduce time to treatment in various health care systems across the world. A prior meta-analysis of randomised trials of in-hospital versus prehospital fibrinolysis found a 58 minute reduction in time to treatment with associated mortality benefit to patients treated in the prehospital setting (30 day mortality relative risk reduction $17 \%$, absolute risk reduction $1.7 \%, p=0.03) .{ }^{3}$ Since then the CAPTIM (comparison of angioplasty to prehospital thrombolysis trial in myocardial infarction) study showed that primary percutaneous coronary intervention (PCI) has no survival advantage as compared with rapid pharmacological reperfusion in the prehospital setting $(4.8 \% \vee 3.8 \%$, not significant). ${ }^{4}$ In fact when patients were stratified according to treatment within two hours from symptom onset the 30 day survival favoured fibrinolysis $(5.7 \% v 2.2, \mathrm{p}=0.058) .{ }^{4}$

Multiple factors have been cited to explain the limited implementation of prehospital fibrinolysis programmes, especially in North America. ${ }^{1}$ Although paramedics with appropriate education and remote physician support administered fibrinolysis successfully in the MITI (myocardial infarction triage and intervention) trial, the lack of physicians in the prehospital setting in many countries remains a perceived barrier. ${ }^{6}$ The ASSENT (assessment of the safety and efficacy of a new thrombolytic) -3 PLUS randomised trial in acute myocardial infarction enrolled 1639 patients with STEMI within six hours in 12 countries who were treated with prehospital fibrinolysis. ${ }^{7}$ Sites participating in this trial operated with different models, including whether a physician was present in the prehospital setting and with various protocols for assessing, diagnosing, and treating patients with STEMI. As the largest contemporary prehospital fibrinolysis trial, ASSENT-3 PLUS offers a unique opportunity to compare the process and outcomes associated with prehospital fibrinolysis across international boundaries. The objectives of the current prehospital study were, firstly, to

Abbreviations: ASSENT, assessment of the safety and efficacy of a new thrombolytic; CAPTIM, comparison of angioplasty to prehospital thrombolysis trial in myocardial infarction; EMS, emergency medical services; ER-TIMI-23, early retavase-thrombolysis in myocardial infarction; MITI, myocardial infarction triage and intervention; $\mathrm{PCl}$ percutaneous coronary intervention; PRAGUE-2, primary angiography in patients transferred from general community hospitals to specialized PTCA units with or without emergency thrombolysis; STEMI, ST elevation myocardial infarction 
assess the predictors of time to treatment in patients with STEMI; secondly, to assess whether the components of time to treatment varied across participating countries; and thirdly, to evaluate the impact of a physician's presence before the patient's hospitalisation on time to treatment, adherence to protocol, and clinical events.

\section{METHODS}

ASSENT-3 PLUS randomly assigned patients in the prehospital setting to treatment with tenecteplase in combination with unfractionated heparin or the low molecular weight heparin enoxaparin. Tenecteplase is a single bolus weight adjusted fibrinolytic that is equivalent to alteplase with respect to mortality with an enhanced safety profile. ${ }^{8}$ Unfractionated heparin was administered according to American College of Cardiology/American Heart Association guidelines with an intravenous bolus of $60 \mathrm{U} / \mathrm{kg}$ (maximum of $4000 \mathrm{U}$ ) and an initial infusion of $12 \mathrm{U} / \mathrm{kg}$ (maximum of $1000 \mathrm{U} / \mathrm{h}) .{ }^{9}$ Prehospital teams were given the option of initiating the intravenous infusion of unfractionated heparin on arrival at hospital if the time from administration of the bolus to hospital arrival was perceived to be less than 30 minutes. Enoxaparin was administered as an initial intravenous bolus of $30 \mathrm{mg}$ simultaneously with a subcutaneous injection of $1 \mathrm{mg} / \mathrm{kg}$ (maximum of $100 \mathrm{mg}$ for first two doses). The median time from symptom onset until initiation of treatment with prehospital fibrinolysis was 115 minutes (53\% of patients were treated within two hours) and 47 minutes earlier than the contemporary ASSENT-3 trial of inhospital fibrinolysis with identical inclusion and exclusion criteria. $^{7}$ Although enoxaparin in ASSENT-3 PLUS tended to reduce the composite end point of 30 day mortality, inhospital reinfarction, or in-hospital refractory ischaemia, there was an associated increased risk in total stroke and intracranial haemorrhage, specifically in patients older than 75 years.

Multiple logistic regression analysis was undertaken to determine patient characteristics related to early treatment in two hours or less from symptom onset, which approximated the median time to treatment in the trial. The adjustment was based on all the baseline characteristics listed in table 2, but only age, heart rate, systolic blood pressure, and prior PCI remained as independent predictors in the model. In the comparison of international variation in time to treatment, countries that enrolled at least 50 patients were analysed individually (1593 of 1639 patients) with the remaining countries grouped together (other). Variation in the process whereby prehospital fibrinolysis was administered across regional and international boundaries was anticipated in ASSENT-3 PLUS, which involved 106 centres in 12 countries. Therefore, a comprehensive prehospital "process of care" survey was developed, validated, and distributed to all active study sites to assess aspects of prehospital care in various health care environments participating in the trial. ${ }^{10}$ The survey had a $99 \%$ return rate and responding sites accounted for $95 \%$ of patients enrolled in ASSENT-3 PLUS. To assess the impact of a physician's presence before hospitalisation, patient data were linked with site specific survey responses. When data were missing regarding the presence or absence of a physician in the emergency medical services (EMS) vehicle (ambulance or mobile intensive care unit) verification and imputation were undertaken based on the established practice in that country ( $\mathrm{n}=160$ patients). Masquerading myocardial infarction was analysed based on 1541 patients, since 98 were missing required data for analysis (44 dead within a day, 45 missing baseline ECG, and nine missing other data). Masquerading myocardial infarction was defined as ST segment elevation on the baseline ECG that does not resolve in conjunction with no increase of myocardial enzymes greater than two times the upper limit of normal. ${ }^{11}$ Results are presented as medians and percentages unless otherwise stated. Logistic regression analysis results are presented as the calculated odds ratio with $95 \%$ confidence intervals. Significance was defined a priori at the 0.05 level.

\section{RESULTS \\ Patient characteristics associated with earlier treatment ( $\leqslant 2$ hours)}

Younger age, slower heart rate, lower systolic blood pressure, and prior PCI predicted treatment in two hours or less (fig l). When the country of enrolment was included in the multiple logistic regression model, it was found to account for the largest proportion $(47 \%)$ of the variation in time to treatment.

\section{International variation in time to treatment}

There was a significant interaction of time to treatment and patient country of origin $(\mathrm{p}<0.001)$. Median time (hours:minutes) to treatment was longer in France (2:05), Spain (2:05), and the UK (2:01). It was shorter in Finland (1:30), Germany (1:35), the Netherlands (1:35), Canada (1:38), and Sweden (1:50). The different components of time to treatment were variable (fig 2). Time from symptom onset to first call for medical assistance was shortest in Finland

\begin{tabular}{lccc} 
Variable & $\mathrm{p}$ & $\mathrm{OR}$ & $95 \% \mathrm{Cl}$ \\
\hline Age (10 years) & 0.001 & 1.22 & $(1.12$ to 1.32$)$ \\
$\mathrm{HR}(10$ beats $/ \mathrm{min})$ & $<0.01$ & 1.12 & $(1.06$ to 1.19$)$ \\
$\mathrm{SBP}(10 \mathrm{~mm} \mathrm{Hg})$ & 0.011 & 1.06 & $(1.01$ to 1.10$)$ \\
Past PCl & 0.049 & 0.66 & $(0.44$ to 1.00$)$ \\
Anterior $\mathrm{Ml}$ & 0.122 & 0.85 & $(0.69$ to 1.05$)$ \\
Female & 0.181 & 1.19 & $(0.92$ to 1.53$)$ \\
Past CABG & 0.436 & 1.33 & $(0.65$ to 2.72$)$
\end{tabular}

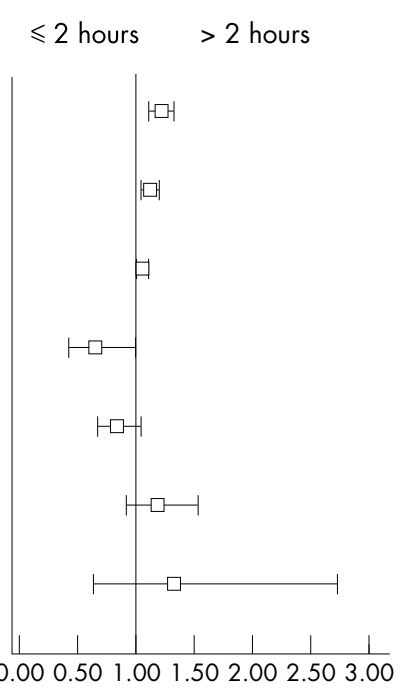

Figure 1 Odds ratio (OR) with 95\% confidence intervals $(\mathrm{Cl})$ and $p$ values of clinical predictors of earlier treatment measured as symptom onset to initiation of fibrinolysis $\leqslant 2$ hours. Anterior MI, anterior wall ST elevation myocardial infarction; $C A B G$, coronary artery bypass grafting; HR, heart rate; $\mathrm{PCl}$, percutaneous coronary intervention, SBP, systolic blood pressure. 


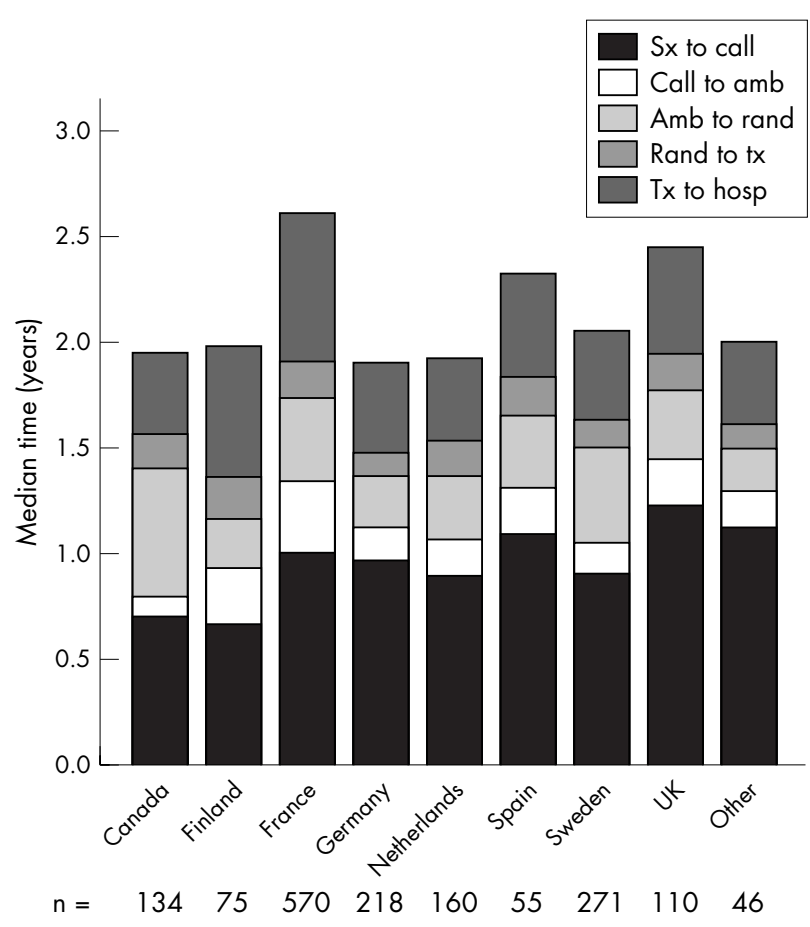

Figure 2 Median time to treatment for all participating countries that enrolled at least 50 patients with the remaining countries combined as other (Belgium, Italy, Norway, USA). Sx to call, patient symptom onset until calling emergency medical services (EMS) for assistance; Call to amb, call to EMS vehicle (ambulance/medical intensive care unit) arrival to the patient; Amb to rand, EMS vehicle arrival until randomisation in the study; Rand to tx, randomisation until administration of tenecteplase; $T x$ to hosp, time from administration of tenecteplase until arrival at receiving hospital.

(40 minutes) and longest in the UK (74 minutes). Time from patient call for assistance to EMS vehicle arrival on scene was shortest in Canada (six minutes) and longest in France (20 minutes). Elapsed time from arrival to randomisation was shortest in Finland and Germany (14 minutes) and longest in Canada (36 minutes). Time from randomisation to treatment was rapid and homogeneous across countries. The subsequent on-scene time before hospital arrival after administration of reperfusion, which was longest in France, was heterogeneous.

Table 1 ASSENT-3 PLUS national patient enrolment, number of study sites, and presence or absence of physician in the prehospital setting

\begin{tabular}{lll}
\hline Country & $\begin{array}{c}\text { Patients } \\
\text { enrolled }\end{array}$ & $\begin{array}{l}\text { Physician present } \\
\text { prehospitalisation* }\end{array}$ \\
\hline France & 570 & $100 \%(18 / 18)$ \\
Sweden & 271 & $0 \%(0 / 14)$ \\
Germany & 218 & $100 \%(13 / 13)$ \\
The Netherlands & 160 & $0 \%(0 / 3)$ \\
Canada & 134 & $0 \%(0 / 3)$ \\
UK & 110 & $60 \%(3 / 5)$ \\
Finland & 75 & $100 \%(1 / 1)$ \\
Spain & 55 & $100 \%(10 / 10)$ \\
Belgium & 20 & $100 \%(3 / 3)$ \\
Italy & 15 & $100 \%(5 / 5)$ \\
Norway & 10 & $50 \%(1 / 2)$ \\
USA & 1 & $0 \%(0 / 1)$ \\
\hline *Number of sites with a physician divided by total number of sites.
\end{tabular}

International variation in prehospital process of care: presence or absence of a physician

In France, Germany, Finland, Spain, Belgium, and Italy physicians participated in the prehospital care of patients with STEMI (table 1). In Sweden, the Netherlands, and Canada prehospital care was delivered by an advanced cardiac life support trained EMS paramedic or nurse. The UK and Norway had sites with and without a physician in the prehospital setting. Of the total study population a prehospital physician managed 63.8\% (1045 of 1639).

\section{Time to treatment}

Median time to treatment was longer in study sites with a physician present in the prehospital environment (120 v 108 minutes, $p<0.001$ ). These sites had a longer time from patient call for assistance to EMS vehicle arrival, a shorter time from arrival to randomisation, and a longer time from initiation of treatment until hospital arrival (fig 3).

\section{Clinical events}

Differences in patient characteristics were observed between sites with or without a prehospital physician (table 2). Among sites with a physician present, patients were younger, more likely to be male, and more likely to have a history of hypertension but less likely to have prior coronary artery bypass surgery. Table 3 presents event rates in patients managed with or without a physician. Patients enrolled at sites with a prehospital physician had more intracranial haemorrhage (adjusted 2.0\% v 0.8\%, p = 0.034). Prehospital and in-hospital bleeding complications (composite of major and serious bleeding, stroke, and intracranial haemorrhage) were not different between the two groups (5.6\% $v 6.2 \%$, $\mathrm{p}=0.584)$.

\section{Adherence to protocol}

Misdiagnoses as assessed by the rate of masquerading myocardial infarction were not different with the presence or absence of a physician (2.7\% v 3.4\%, not significant). Physicians in the prehospital setting more thoroughly completed required documentation and obtained study data as measured by fewer missing baseline ECGs $(2.5 \% v 4.5 \%$, $\mathrm{p}=0.029)$ and more complete recording of prehospital Killip class $(97.6 \% v 46.5 \%, \mathrm{p}<0.001)$.

In patients treated with versus without a physician before hospitalisation, respectively, the administered dose of tenecteplase tissue plasminogen activator was correct (95-105\%) in $75.8 \%$ versus $75.6 \%(\mathrm{p}=0.952)$, greater than $105 \%$ in $21.0 \%$ versus $18.8 \%(\mathrm{p}=0.301)$, and less than $95 \%$ in $3.2 \%$ versus $5.7 \%(p=0.019)$. No relation was documented between the dose of tenecteplase tissue plasminogen activator administered $(95-105 \%,>105 \%$ or $<95 \%)$ with any of the efficacy end points (death, repeat MI, or refractory ischaemia). Administration of the initial bolus dose of study anticoagulation medication, unfractionated heparin or enoxaparin, was not different according to physician's presence (>99\% of all patients). Intravenous infusion of unfractionated heparin or subcutaneous injection of enoxaparin was administered to $98.0 \%$ of patients with a physician and $98.6 \%$ of those without $(p=0.436)$; however, initiation of the heparin infusion until arrival at the hospital was delayed much less often in the presence of a physician $(16.5 \% v$ $42.1 \%, \mathrm{p}<0.001$ ). Risk of death, reinfarction, or refractory ischaemia did not increase with this delayed initiation of the intravenous infusion. Study anticoagulation was discontinued due to adverse events less often in patients enrolled with a physician $(20.2 \% \vee 32.8 \%, \mathrm{p}<0.001)$. 


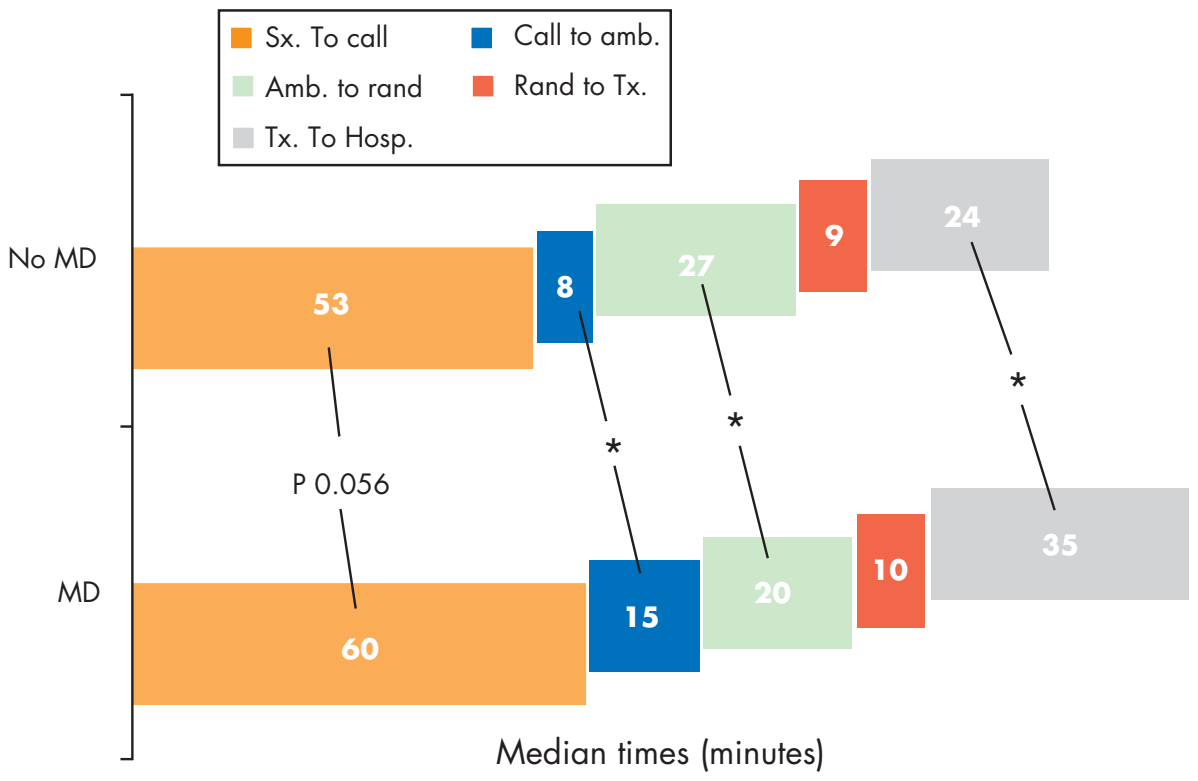

Figure 3 Median times of each component of time to treatment in enrolled patients with or without a physician present before hospitalisation. * $p<0.05$ between components across groups according to presence of a physician.

In-hospital and subsequent patient management To address potential confounding factors after the transition from prehospital to in-hospital patient care, differences in patient management in hospital in sites with or without a prehospital physician are presented (table 4).

\section{DISCUSSION}

This study from the largest contemporary prehospital fibrinolysis trial, ASSENT-3 PLUS, provides unique information on the process and outcomes associated with prehospital fibrinolysis across international boundaries in diverse health systems. Earlier time to treatment was achieved for patients who were younger and who had a lower heart rate, lower systolic blood pressure, and prior PCI. The country of enrolment accounted for the largest proportion of variation in time to treatment and components of delay were heterogeneous. The presence of a physician in the prehospital setting was associated with greater adherence to protocol mandated treatments and procedures but also with delay in time to treatment.

Although regional and international conditions vary, about half of patients with STEMI present to medical attention through prehospital EMS. ${ }^{12}{ }^{13}$ The current study provides a contemporary assessment of predictors of treatment delay specific to this subset of patients who have activated the EMS system and subsequently received prehospital fibrinolysis.
Our results showed a prolonged time to treatment with older patients, which is consistent with prior studies of in-hospital fibrinolysis. ${ }^{14-16}$ A prior history of coronary artery disease has been associated with delayed treatment but prior PCI was associated with earlier treatment in this prehospital fibrinolysis study..$^{1416}$ Patient education after PCI is possibly associated with earlier presentation as well as increased probability of contacting the EMS system for medical assistance in the face of recurrence of symptoms, which has been recently reported. ${ }^{17}$ Past research has shown that female STEMI patients are more likely to present to hospital by ambulance but conversely have delayed time from symptom onset to in-hospital fibrinolysis, which is consistent with our results. ${ }^{14} 16$

Although international variation in management of STEMI patients has been observed, this is the first study to show that the country of enrolment accounts for the largest proportion of variation in time to treatment with prehospital fibrinolysis. Delays that occur in the prehospital setting are poorly studied to date and may be related to systematic or procedural aspects of the triage, assessment, and treatment of patients with STEMI by prehospital care providers. Such systematic delays have been reported due to hospital procedures and policy regarding patients who received in-hospital pharmacological reperfusion. ${ }^{12}$ This study tracked multiple temporal aspects specific to prehospital care and as such provides

Table 2 Patient characteristics with or without a physician present before hospitalisation

\begin{tabular}{|c|c|c|c|c|}
\hline & \multirow{2}{*}{$\begin{array}{l}\text { ASSENT-3 } \\
\text { PLUS }\end{array}$} & \multicolumn{2}{|c|}{ Prehospital physician } & \multirow[b]{2}{*}{$p$ Value present $v$ absent } \\
\hline & & Present & Absent & \\
\hline Sample size & 1639 & 1045 & 594 & \\
\hline Age (years)* & 62 & 61 & 64 & $<0.001$ \\
\hline Female sex & $23 \%$ & $20 \%$ & $28 \%$ & 0.001 \\
\hline Current smoking & $43 \%$ & $44 \%$ & $41 \%$ & 0.363 \\
\hline Diabetes & $15 \%$ & $16 \%$ & $13 \%$ & 0.194 \\
\hline Hypertension & $36 \%$ & $40 \%$ & $29 \%$ & $<0.001$ \\
\hline Prior MI & $15 \%$ & $14 \%$ & $16 \%$ & 0.189 \\
\hline Prior $\mathrm{PCl}$ & $7 \%$ & $8 \%$ & $6 \%$ & 0.221 \\
\hline Prior CABG & $2 \%$ & $1 \%$ & $3 \%$ & 0.019 \\
\hline Heart rate (beats $/ \mathrm{min})^{*}$ & 74 & 74 & 73 & 0.148 \\
\hline $\mathrm{SBP}(\mathrm{mm} \mathrm{Hg})^{*}$ & 133 & 135 & 132 & 0.014 \\
\hline
\end{tabular}


Table 3 Clinical events with and without a physician present before hospitalisation

\begin{tabular}{|c|c|c|c|c|c|}
\hline \multirow[b]{2}{*}{ Variable } & \multicolumn{2}{|c|}{ Prehospital physician } & \multirow[b]{2}{*}{ Unadjusted OR $(95 \% \mathrm{CI})$} & \multirow[b]{2}{*}{ Adjusted OR $(95 \% \mathrm{Cl})$} & \multirow[b]{2}{*}{$\mathrm{p}$ Value } \\
\hline & Present & Absent & & & \\
\hline $\begin{array}{l}\text { Sample size } \\
\text { Pre-/in-hospital events }\end{array}$ & 1045 & 594 & & & \\
\hline Refractory ischaemia & 5.4 & 5.6 & $0.96(0.62$ to 1.50$)$ & $0.92(0.59$ to 1.44$)$ & 0.706 \\
\hline Reinfarction & 4.0 & 5.9 & $0.67(0.42$ to 1.06$)$ & $0.66(0.42$ to 1.05$)$ & 0.082 \\
\hline Stroke (in hospital) & 2.5 & 1.5 & $1.66(0.77$ to 3.56$)$ & $2.02(0.92$ to 4.44$)$ & 0.081 \\
\hline $\begin{array}{l}\text { Major bleeding } \\
30 \text { day events }\end{array}$ & 2.8 & 4.5 & 0.60 (0.35 to 1.02$)$ & $0.68(0.39$ to 1.17$)$ & 0.164 \\
\hline Death & 6.7 & 6.7 & $1.00(0.67$ to 1.49$)$ & $1.27(0.82$ to 1.98$)$ & 0.292 \\
\hline Reinfarction & 4.2 & 6.4 & $0.64(0.41$ to 1.01$)$ & $0.68(0.43$ to 1.06$)$ & 0.087 \\
\hline Serious recurrent ischaemia & 1.3 & 2.5 & $0.52(0.25$ to 1.09$)$ & 0.51 (0.25 to 1.07$)$ & 0.077 \\
\hline Intracranial haemorrhage & 2.0 & 0.8 & 2.42 (0.91 to 6.44$)$ & 2.99 (1.08 to 8.23$)$ & 0.034 \\
\hline Death/repeat MI & 10.2 & 12.8 & $0.77(0.57$ to 1.06$)$ & $0.88(0.63$ to 1.23$)$ & 0.451 \\
\hline Death/Ml/ischaemia & 11.2 & 15.2 & $0.71(0.53$ to 0.95$)$ & 0.80 (0.58 to 1.09$)$ & 0.148 \\
\hline
\end{tabular}

insight into potential intracountry systemic delays. Consistent with past research, the time from symptom onset until first medical contact accounts for the majority of delay in time to treatment, but this study shows substantial heterogeneity across participating countries. In the UK, which had the longest delay to activation of EMS, a policy that requires contact of a primary care physician who subsequently activates EMS possibly establishes a systematic delay. The time from activation of EMS to arrival on scene was longest in France, which may be related to systematic delay arising from selective triage of a limited number of physician manned EMS units (medical intensive care unit) to the care of patients with chest pain or secondary dispatch of such units after preliminary assessment by non-physician staffed units. Alternatively the realities of urban traffic in large centres such as Paris may contribute to this delayed response. Once EMS have arrived on scene the delay until randomisation was longest in Canada, where paramedic based units assessed patients to determine eligibility, completed study documentation, and transmitted the 12 lead ECG for physician overread with subsequent random assignment of patients. Understanding the heterogeneity in these temporal components in various nations and health

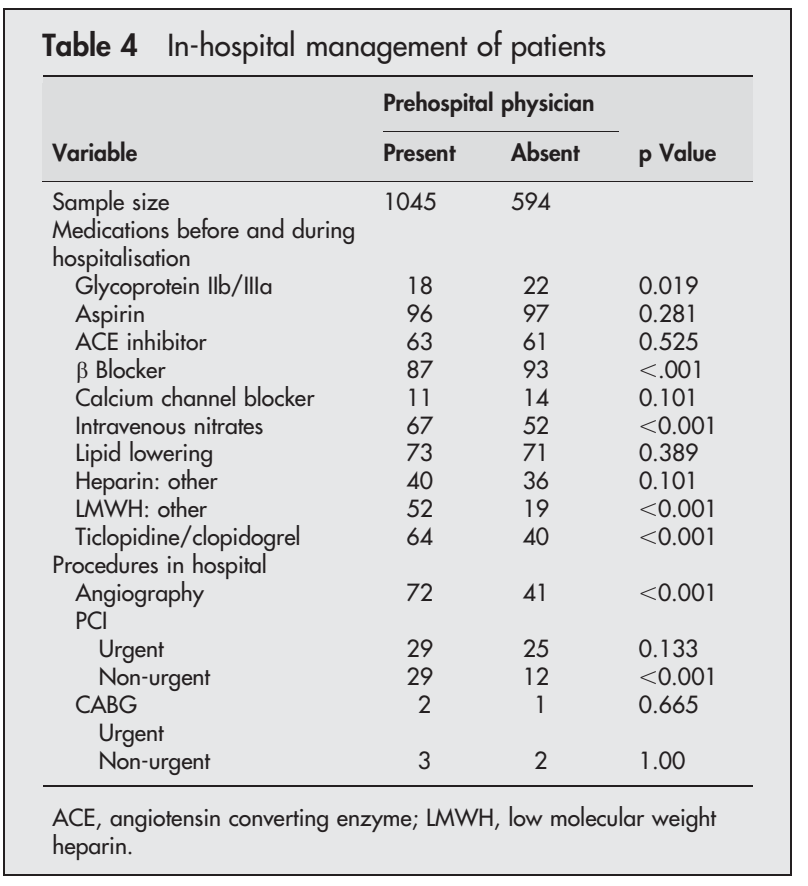

care systems may enhance the opportunities of prehospital fibrinolysis programmes to specifically target the most relevant aspects of delay in treatment with focused resource application maximising benefits in individual countries.

The most striking difference in the process of prehospital care across the countries participating in this study was the presence or absence of physicians. The delay in treatment with a prehospital physician conceivably is related to the number of "capable EMS units". Specifically, physician manned EMS units capable of administering treatment to patients with STEMI are a limited resource and require a triage based dispatch or alternatively non-physician staffed ambulance units operating as primary responders with subsequent dispatch of physician staffed medical intensive care units. Once on scene the prehospital physician more quickly assesses the patient, diagnoses his or her condition, and randomly assigns the patient faster, possibly due to advanced training, clinical experience, and the ability to interpret the 12 lead ECG without transmission. After administering treatment the prehospital team with a physician delayed transport, spending more time on the scene. Since it is not possible in this analysis to separate prolonged on-scene time from prolonged transport time, the increased time from treatment until hospital arrival with a physician before hospitalisation may also be due to a conscious decision to triage the patient to a hospital capable of mechanical co-intervention, which may be at a further distance.

Adherence to study protocol and procedures, and accurate administration of treatment are important elements of research conduct as well as clinical practice. In this study there was no increased risk of misdiagnosis as measured by the rate of masquerading STEMI with or without a physician in the prehospital setting. Other measures of adherence to study protocol, documentation of Killip class and availability of baseline ECGs, appear to have been more diligently completed when a physician was present. Medication errors in hospital are known to be common and to be associated with adverse outcomes and increased length of hospital stay and are estimated to contribute to $44000-98000$ deaths annually in the USA alone. ${ }^{18-20}$ Although the ability of personnel to appropriately measure and administer treatment in the prehospital environment has not been thoroughly assessed, available data suggest that overall paramedical performance is poor. ${ }^{21}$ The presence of a physician in this study was associated with a strong trend to a decreased rate of medication errors in the administration of the bolus fibrinolytic tenecteplase and with more consistent administration of the unfractionated heparin before hospital arrival, 
although there was no attributable increased patient risk. In the treatment of STEMI with complex pharmacological regimens including antiplatelets, anticoagulants, and fibrinolytic agents, the importance of correct administration of medications is paramount and its implications have been discussed.22

The outcomes of patients enrolled in clinical trials or studies that assess the impact of variation in process of care, such as this one, remain important considerations. Although the presence of a physician was associated with a trend to a reduced rate of recurrent ischaemia and repeat myocardial infarction at 30 days, these observations may be confounded by differences in subsequent in-hospital revascularisation. Furthermore, the combined rates of major bleeding, stroke, and intracranial haemorrhage were not different, therefore suggesting the safety of either approach to prehospital care, at least in a clinical trial setting. On balance no significant clinical benefit or risk was associated with the presence or absence of a prehospital physician in ASSENT-3 PLUS. This study is aligned with the results of prior investigations, MITI and ER-TIMI-23 (early retavase-thrombolysis in myocardial infarction), and supports the capability of non-physician based prehospital EMS care providers to administer fibrinolysis with appropriate regional and remote physician support, $^{6}{ }^{23}$ thereby encouraging regional investment in prehospital fibrinolysis programmes.

Because our study is based on a non-randomised assessment of variation in process of care across international boundaries in selected centres participating in ASSENT 3PLUS, it may not be generally representative of the standard of prehospital care in the respective country and therefore has inherent limitations. The combination of patient data with site specific survey responses to assess the impact of variation in prehospital care on patient treatment and outcomes may be influenced by investigator biases. The assessment of the presence or absence of a physician is potentially confounded by the associated medical system in which the patients were enrolled and the subsequent in-hospital management. Furthermore, the lack of a comprehensive registry of all patients with STEMI in regions participating in the trial does not allow assessment of potential patients who were missed by the prehospital teams.

International registry data have shown that only $41 \%$ of patients who receive in-hospital fibrinolysis present to hospital within two hours of symptom onset and only one third receive treatment within two hours. ${ }^{24}$ In contrast patients who activate the EMS system and subsequently receive prehospital fibrinolysis achieved a median time to treatment of two hours in ASSENT-3 PLUS, which is similar to that achieved in the fibrinolysis arm of the CAPTIM trial. ${ }^{57}$ A post hoc analysis of the PRAGUE-2 (primary angiography in patients transferred from general community hospitals to specialized PTCA units with or without emergency thrombolysis) study has shown similar efficacy of in-hospital streptokinase and primary PCI in patients treated within three hours of symptom onset. ${ }^{25}$ The integration of these findings-that median time to treatment in prehospital fibrinolysis trials approximates two hours and that patients who receive pharmacological reperfusion early (2-3 hours) have excellent outcomes as good as or superior to primary PCI-provides further impetus to advance prehospital fibrinolysis programs.

\section{Conclusion}

Time to treatment with prehospital fibrinolysis varies internationally. The presence of a physician before hospitalisation in many international health care systems is perceived as impractical and a limitation to widespread implementation of prehospital fibrinolysis programmes.
Although aspects of prehospital care and the management of complex patients who do not fit trial criteria are conceivably enhanced by the presence of an experienced physician, in ASSENT-3 PLUS prehospital fibrinolysis administered by a physician or trained paramedical staff resulted in significant reduction in time to treatment in comparison with ASSENT-3. Moreover, the effectiveness and safety of prehospital fibrinolysis was not influenced by the presence of a physician. The current study expands our understanding of the systems required to administer treatment before hospital arrival and provides encouragement for stakeholders committed to enhancing care of STEMI patients.

\section{Authors' affiliations}

R C Welsh, W Chang, P W Armstrong, University of Alberta, Edmonton, Alberta, Canada

P Goldstein, SAMU Regional de Lille Centre Hospitalier de Lille, Lille,

France

J Adgey, Royal Victoria Hospital, Belfast, UK

C B Granger, Division of Cardiology, Duke Clinical Research Institute, Durham, North Carolina, USA

F W A Verheugt, Cardiology University Medical Centre St Radboud, Nijmegen, the Netherlands

L Wallentin, Cardiology University Hospital, Uppsala, Sweden

F Van de Werf, Department of Cardiology, University Hospital Gasthuisberg, Leuven, Belgium

The data in this manuscript have never been published in any other form. The project was funded by a grant in aid from Boehringer Ingelheim, Aventis Inc, and Hoffman-La Roche Canada.

\section{REFERENCES}

1 Welsh RC, Ornato J, Armstrong PW. Prehospital management of acute STelevation myocardial infarction: a time for reappraisal in North America. Am Heart J 2003;145:1-8.

2 Rogers WJ, Canto JG, Lambrew CT, et al. Temporal trends in the treatment of over 1. 5 million patients with myocardial infarction in the US from 1990 through 1999: the national registry of myocardial infarction 1, 2 and 3. J Am Coll Cardiol 2000;36:2056-63.

3 Morrison LJ, Verbeek PR, McDonald AC, et al. Mortality and prehospital thrombolysis for acute myocardial infarction: a meta-analysis. JAMA 2000;283:2686-92

4 Bonnefoy E, Lapostolle F, Leizorovicz A, et al. Primary angioplasty versus prehospital fibrinolysis in acute myocardial infarction: a randomised study. Lancet 2002;360:825-9.

5 Steg PG, Bonnefoy E, Chabaud S, et al. Impact of time to treatment on mortality after prehospital fibrinolysis or primary angioplasty: data from the CAPTIM randomized clinical trial. Circulation 2003;108:2851-6.

6 Weaver WD, Cerqueira M, Hallstrom AP, et al. Prehospital-initiated vs hospital-initiated thrombolytic therapy. The myocardial infarction triage and intervention trial. JAMA 1993;270:1211-6.

7 Wallentin L, Goldstein P, Armstrong PW, et al. Efficacy and safety of tenecteplase in combination with the low-molecular-weight heparin enoxaparin or unfractionated heparin in the prehospital setting: the assessment of the safety and efficacy of a new thrombolytic regimen (ASSENT)-3 PLUS randomized trial in acute myocardial infarction. Circulation 2003; 108: 135-42.

8 ASSENT-2 Investigators. Single-bolus tenecteplase compared with frontloaded alteplase in acute myocardial infarction: the ASSENT-2 double-blind randomised trial, Assessment of the safety and efficacy of a new thrombolytic investigators. Lancet 1999:354:716-22.

9 Ryan TJ, Antman EM, Brooks NH, et al. 1999 update: ACC/AHA guidelines for the management of patients with acute myocardial infarction: executive summary and recommendations: a report of the American College of Cardiology/American Heart Association task force on practice guidelines (committee on management of acute myocardial infarction). Circulation 1999; 100:1016-30.

10 Welsh RC, Goldstein P, Adgey J, et al. Variations in pre-hospital fibrinolysis process of care: insights from the assessment of the safety and efficacy of a new thrombolytic 3 PLUS international acute myocardial infarction prehospital care survey. Eur J Emerg Med 2004;11:134-40.

11 Taher T, Fu Y, Wagner GS, et al. Aborted myocardial infarction in patients with ST segment elevation: insights from the assessment of the safety and efficacy of a new thrombolytic regimen-3 trial electrocardiographic substudy. J Am Coll Cardiol 2004;44:38-43.

12 Lambrew CT, Bowlby $\amalg$, Rogers WJ, et al. Factors influencing the time to thrombolysis in acute myocardial infarction. Time to thrombolysis substudy of the national registry of myocardial infarction-1. Arch Intern Med 1997; 157:2577-82.

13 Hirvonen TP, Halinen MO, Kala RA, et al. Delays in thrombolytic therapy for acute myocardial infarction in Finland: results of a national thrombolytic 
therapy delay study. Finnish hospitals' thrombolysis survey group. Eur Heart $J$ 1998:19:885-92.

14 Berglin BM, Hartford M, Karlsson T, et al. Factors associated with pre-hospital and in-hospital delay time in acute myocardial infarction: a 6-year experience. J Intern Med 1998;243: 243-50.

15 Berton G, Cordiano R, Palmieri R, et al. Clinical features associated with prehospital time delay in acute myocardial infarction. Ital Heart $J$ 2001;2:766-71.

16 Leizorovicz A, Haugh MC, Mercier C, et al. Pre-hospital and hospital time delays in thrombolytic treatment in patients with suspected acute myocardial infarction: analysis of data from the EMIP study. European myocardial infarction project. Eur Heart $J$ 1997:18:248-53.

17 Cabrita B, Bouyer-Dalloz F, L'Huillier I, et al. Beneficial effects of direct call to emergency medical services in acute myocardial infarction. Eur J Emerg Med 2004; 11:12-8

18 Bates DW, Cullen DJ, Laird N, et al. Incidence of adverse drug events and potential adverse drug events: implications for prevention. ADE prevention study group. JAMA 1995;274:29-34.
19 Bates DW, Spell N, Cullen DJ, et al. The costs of adverse drug events in hospitalized patients. Adverse drug events prevention study group. JAMA 1997;277:307-11.

20 SoRelle R. Reducing the rate of medical errors in the United States. Circulation 2000;101:E39-40.

21 Hubble MW, Paschal KR, Sanders TA. Medication calculation skills of practicing paramedics. Prehosp Emerg Care 2000;4:253-60.

22 Cannon CP. Thrombolysis medication errors: benefits of bolus thrombolytic agents. Am J Cardiol 2000;85:17C-22C.

23 Morrow DA Antman EM, Sayah A et al. Evaluation of the time saved by prehospital initiation of reteplase for ST-elevation myocardial infarction: results of the early retavase-thrombolysis in myocardial infarction (ER-TIMI) 19 trial. J Am Coll Cardiol 2002;40:71-7.

24 Goldberg RJ, Steg PG, Sadiq I, et al. Extent of, and factors associated with, delay to hospital presentation in patients with acute coronary disease (the GRACE registry). Am J Cardiol 2002;89:791-6.

25 Widimsky P, Budesinsky T, Vorac D, et al. Long distance transport for primary angioplasty vs immediate thrombolysis in acute myocardial infarction: final results of the randomized national multicentre trial-PRAGUE-2. Eur Heart $J$ 2003;24:94-104.

\section{IMAGES IN CARDIOLOGY}

\section{Successful percutaneous closure of acquired atrial septal defect in a man with postoperative pericardial constriction}

\begin{abstract}
$\mathrm{A}^{7}$ 70 year old man was admitted with signs of severe right heart failure 10 months following a mitral valve repair (quadrangular resection and Carpentier ring insertion). Examination revealed a soft systolic murmur and extensive peripheral oedema which responded initially to high doses of furosemide and spironolactone. Transoesophageal echocardiography demonstrated only mild mitral regurgitation and good left ventricular function. Surprisingly there were two defects in the intra-atrial septum (panel A) which had not been present at two previous transoesophageal echocardiograms. At cardiac catheterisation, the left to right shunt was calculated at 1.7:1; however, this was likely to be an underestimation as there was also found to be diastolic equalisation of pressures suggesting pericardial constriction (panel B). It was uncertain whether the left to right shunt or the cardiac constriction was the most likely cause of the patient's symptoms.

The patient initially underwent successful percutaneous closure of the defect in the intra-atrial septum with an Amplatzer Cribiform occluder (panels C, D). It had been the intension to then send the patient for surgical pericardectomy; however, after closure of the atrial defect the patient made a dramatic recovery, presumably due to reduction in the size of the right ventricle and relative relief of the constriction.

The aetiology of the acquired defect in the atrial septum is uncertain but may have been caused by stretch of an existing patent foramen ovale or atrial septal defect, although surgical trauma at the time of mitral valve repair cannot be excluded.
\end{abstract}

S J Leslie

P S Mankad

D B Northridge

s.j.leslie@ed.ac.uk

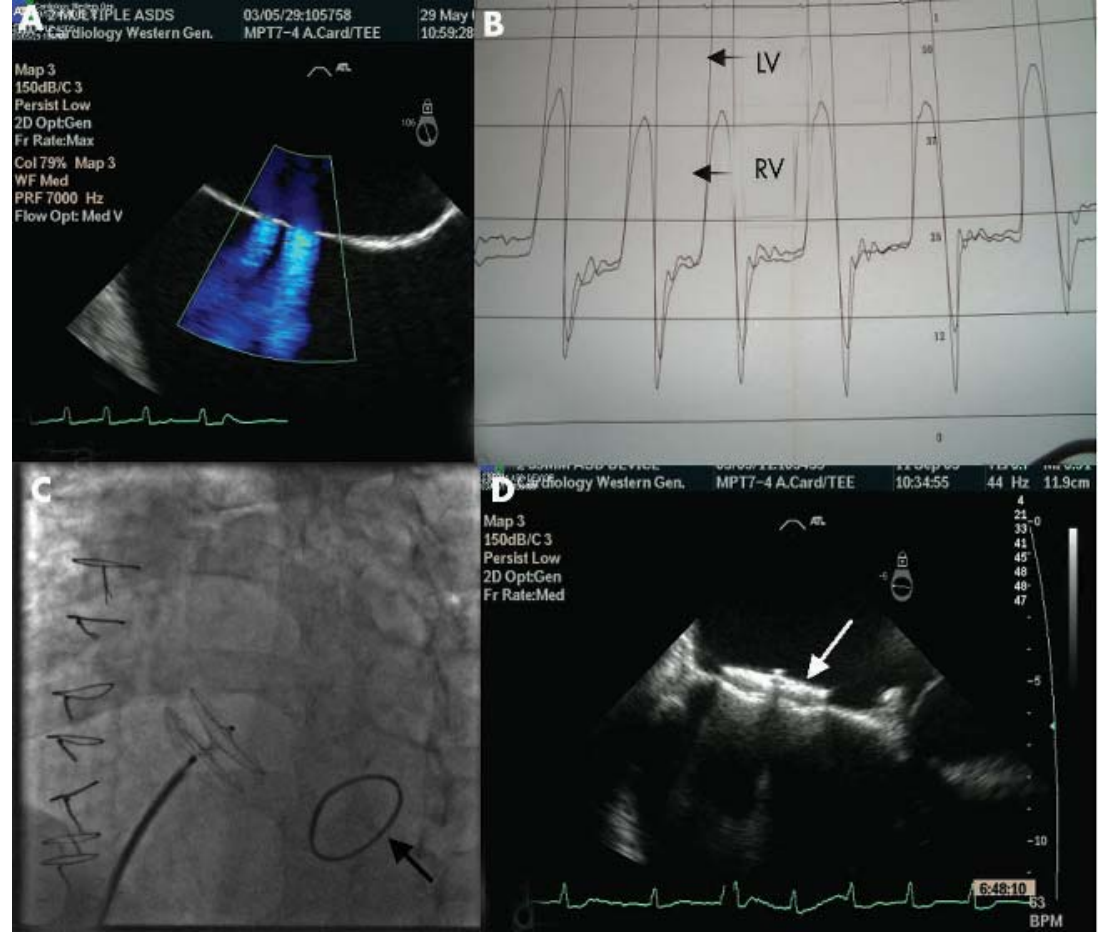

